\title{
Nanoemulsions loaded with amphotericin B: A new approach for the treatment of leishmaniasis
}

\author{
Leila Rodrigues Caldeira ${ }^{\mathrm{a}}$, Flaviana Ribeiro Fernandes ${ }^{\mathrm{b}}$, Daniel Ferreira Costa ${ }^{\mathrm{a}}$, Frédéric Frézard ${ }^{\mathrm{b}}$, \\ Luís Carlos Crocco Afonso ${ }^{\mathrm{c}}$, Lucas Antônio Miranda Ferreira ${ }^{\mathrm{a}, *}$ \\ a Department of Pharmaceutics, Faculty of Pharmacy, Federal University of Minas Gerais (UFMG), Av. Antônio Carlos, 6627, 31270-901 Belo Horizonte, Minas Gerais, Brazil \\ ${ }^{\mathrm{b}}$ Department of Physiology and Biophysics, Institute of Biological Sciences, Federal University of Minas Gerais (UFMG), Av. Antônio Carlos, 6627, $31270-901$ Belo Horizonte, \\ Minas Gerais, Brazil

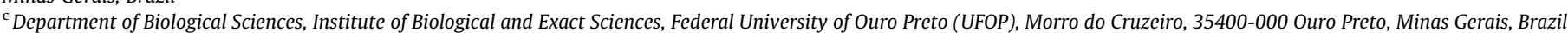

\section{A R T I C L E I N F O}

\section{Article history:}

Received 20 October 2014

Received in revised form 7 January 2015

Accepted 27 January 2015

Available online 4 February 2015

\section{Keywords:}

Amphotericin B

Nanoemulsion

Stearylamine

Cholesterol

Cytotoxicity

Antileishmanial activity

\begin{abstract}
A B S T R A C T
This work aimed to develop nanoemulsions (NE) containing cholesterol and Amphotericin B (AmB) evaluating the influence of a lipophilic amine (stearylamine; STE) on drug encapsulation efficiency (EE), cytotoxicity on macrophages and in vitro antileishmanial activity. The EE of AmB in NE was nearly $100 \%$ regardless of STE concentration. Stability studies showed that AmB-loaded NE with or without STE were stable revealing that $\mathrm{AmB}$ content and $\mathrm{EE}$ remained constant after 180 days. In significant contrast, the EE for AmB in NE without cholesterol drastically decreased showing that this co-surfactant significantly improved the retention of drug in NE. The electronic absorption and circular dichroism (CD) data revealed that the signal characteristic of self-associated free AmB, the most toxic form to the host cells, was virtually absent in the spectra of AmB-loaded NE. In agreement, NE-induced toxicity toward macrophages was significantly lower than that observed for the conventional AmB. STE enhanced both cytotoxicity and the activity against intracellular amastigotes of AmB-loaded NE. However, selectivity index values for AmB-loaded NE were considerably higher than that observed for conventional AmB. AmB-loaded and cholesterol-stabilized NE constitutes an attractive alternative for the treatment of leishmaniasis.

(c) 2015 Elsevier B.V. All rights reserved.
\end{abstract}

\section{Introduction}

Leishmaniasis is a disease with a wide spectrum of clinical manifestations caused by different species of protozoa belonging to the Leishmania genus. Parenteral administration of pentavalent antimony organic compounds remains as the first choice therapy for all leishmaniasis forms (Desjeux, 2004). However, resistance and high frequency of side effects are still relevant problems associated with this treatment. Amphotericin B (AmB), a highly hydrophobic drug, is a useful second-line therapy for treatment of leishmaniasis. The drug is highly active, but its clinical use is limited by its frequent toxicity mainly related to the parenteral administration of the conventional formulation, which is composed of mixed micelles of AmB and sodium deoxycholate.

The lipid formulations such as liposomal AmB (Ambisome ${ }^{\circledR}$ ), Aldecet $^{\circledR}$ and Amphocil ${ }^{\circledR}$ appear as alternatives to overcome this

\footnotetext{
* Corresponding author at: Department of Pharmaceutics, Faculty of Pharmacy, Av. Antônio Carlos, 6627, Campus Pampulha - UFMG, 31270-901 Belo Horizonte, Minas Gerais, Brazil. Tel.: +55 313499 6939; fax: +55 3134996830 .

E-mail address: lucas@farmacia.ufmg.br (L.A.M. Ferreira).
}

drawback (Larabi et al., 2003; Torrado et al., 2008; Vyas and Gupta, 2006). It is well known that such systems reduce AmBrelated toxicity, but their high cost and difficulty of manufacturing represent limitations for their widespread use. Therefore, the design of nanocarriers loaded with AmB remains an open question. Nanoemulsions (NE) are attractive carriers of $A m B$, since they are lipid systems that are able to reduce drug-related toxicity, in addition to being inexpensive and easy to manufacture. However, incorporation of $\mathrm{AmB}$ in these systems remains a challenge (Egito et al., 1996; Muller et al., 2004).

Lance et al. (1995) proposed a strategy for incorporation of AmB in the NE, without the use of organic solvents, by employing the $\mathrm{pH}$-solubility profile of the drug and, more specifically, its higher water-solubility at basic $\mathrm{pH}$. This method is simple and easy to prepare AmB-loaded emulsions. However, these authors reported significant changes in particle size of AmB-loaded emulsions after 7 days, showing that they were very unstable. In addition, the potential of such systems for the treatment of leishmaniasis was not reported.

We previously reported the design of NE loaded with AmB using the pH-solubility profile (Santos et al., 2012). The formation of an 
ion pairing between AmB and stearylamine (STE), a lipophilic amine that presents antileishmanial activity (Banerjee et al., 2008; Pal et al., 2004) was proposed as an alternative to improve the stability of NE loaded with AmB. Their potential for the topical treatment of cutaneous leishmaniasis was investigated by conducting permeation studies with damaged pig skin. However, data revealed that dermal penetration of $\mathrm{AmB}$ from NE was low.

In the present study, the potential of these NE for the intravenous treatment of leishmaniasis was investigated, through evaluation of their activity against intracellular amastigotes and cytotoxicity on macrophages. Considering that the state of molecular aggregation of $\mathrm{AmB}$ dictates the toxicity of the final formulation (Egito et al., 2002; Silva et al., 2013), mainly after intravenous administration, discriminatory techniques such as UV-visible spectroscopy and circular dichroism (CD) spectra were used in this investigation. On the other hand, interactions between $\mathrm{AmB}$ and cholesterol molecules have been previously reported as an alternative to control release and to improve stability of lipid nanocarries (Torrado et al., 2008; Vakil and Kwon, 2008). Therefore, this approach was reported here for NE.

\section{Materials and methods}

\subsection{Materials}

Amphotericin B (AmB; Fig. 1a) and conventional AmB (C-AmB; Anforicin $B^{\circledR}$ ), a complex of AmB and sodium desoxycholate, were kindly provided by Cristalia Produtos Químicos Farmacêuticos Ltda (Sao Paulo, Brazil). Medium chain triglycerides (MCT) and polysorbate 80 (Tween $80^{\circledR}$ ) were kindly provided by Croda Brazil Ltda (São Paulo, Brazil). Cholesterol, $\alpha$-tocopherol and stearylamine (STE; Fig. 1b) were purchased from Sigma-Aldrich (Missouri, USA). HPLC grade acetonitrile and tetrahydrofuran were purchased from Tedia (Rio Janeiro, Brazil). HPLC grade methanol was purchased from J.T. Baker (EUA). All other chemicals were of analytical reagent grade.

\subsection{Preparation of the $N E$ loaded with $A m B$}

Composition of the AmB-loaded NE was previously described (Santos et al., 2012) but with a modification. Considering the intended use of NE (intravenous administration), cholesterol was used instead of sorbitan monooleate as lipophilic co-surfactant. Briefly (batch $20 \mathrm{~mL}$ ), the oily phase (OP) was composed of $1000 \mathrm{mg}$ of MCT, $300 \mathrm{mg}$ of Tween 80 (as hydrophilic surfactant), $100 \mathrm{mg}(0.5 \% \mathrm{w} / \mathrm{v})$ of cholesterol, STE $(0 \%, 0.1 \%$ or $0.2 \% \mathrm{w} / \mathrm{v})$, and $10 \mathrm{mg}$ of $\alpha$-tocopherol (antioxidant). The aqueous phase (AP) was composed of glycerol (2.25\%; isotonizer) and purified water. The NE was prepared by the hot homogenization method. Both oil and aqueous phases were heated at $70{ }^{\circ} \mathrm{C}$ and then the AP was

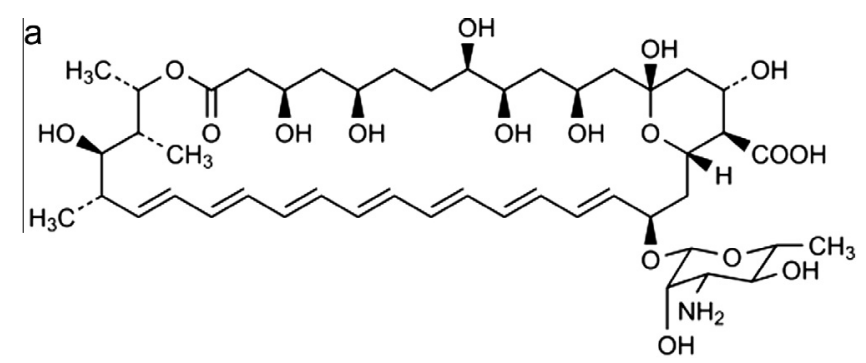

b

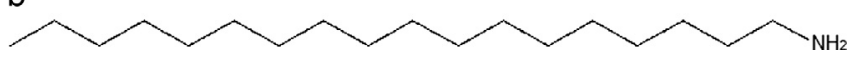

Fig. 1. Chemical structure of AmB and STE. slowly added to the oil phase and homogenized using an Ultra-Turrax T25 at $8000 \mathrm{rpm}$ for $5 \mathrm{~min}$. This emulsion was immediately introduced to the probe sonication (20\% amplitude) for $5 \mathrm{~min}$, using a high intensity ultrasonic processor (Microprocessor controlled, 750 Watt model; Sonics Materials Inc., USA). The AmB was incorporated into the $\mathrm{NE}$ as previously described at a concentration of $2 \mathrm{mg} / \mathrm{mL}$ (Santos et al., 2012). One $\mathrm{mL}$ of a $0.2 \mathrm{M}$ sodium hydroxide $(\mathrm{NaOH})$ solution was added to the blank NE (without $\mathrm{AmB}$ ). Next, this $\mathrm{NE}$ containing $\mathrm{NaOH}$ was cooled to $10^{\circ} \mathrm{C}$. Then, the AmB (40 mg for a batch $20 \mathrm{~mL}$ ) was incorporated into alkaline $\mathrm{NE}$ and the mixture was homogenized using a magnetic stirrer at $600 \mathrm{rpm}$ for $1 \mathrm{~min}$. Next, the NE was immediately removed from the cooling, the $\mathrm{pH}$ was adjusted to 7.0-7.5 with hydrochloric acid at room temperature and finally the final volume was completed with sterile water. A blank NE (without AmB) was also prepared for comparison purpose.

\subsection{Characterization of $N E$ loaded with $A m B$}

Formulations were characterized for particle size (mean hydrodynamic diameter), polydispersity index (PI), zeta potential, $\mathrm{pH}$, AmB content, and drug encapsulation efficiency (EE). UV-visible spectroscopy, circular dichroism (CD) spectra and images of polarized light microscopy were also obtained.

\subsubsection{Determination of droplet size, PI and zeta potential}

The droplet size distribution and the PI were determined by photon correlation spectroscopy (PCS) using a Zetasizer 3000 MHS (Malvern Instruments, UK) at a fixed angle of $90^{\circ}$ and at $25^{\circ} \mathrm{C}$. Before the measurements, the formulations were diluted with distilled water. All measurements were performed in triplicate. Zeta potential was evaluated by determining the electrophoretic mobility using the Zetasizer. Before the measurements, the $\mathrm{NE}$ was diluted in $1 \mathrm{mM} \mathrm{NaCl}$ solution. All measurements were performed in triplicate.

\subsubsection{AmB content and drug encapsulation efficiency (EE)}

The EE of AmB in NE was evaluated based on the determination of AmB concentration before and after filtration (polyvinylidene fluoride membrane, $0.45 \mu \mathrm{m}$ pore size, Millipore ${ }^{\circledR}$, USA) as previously described (Santos et al., 2012). The quantification of AmB before filtration provided the total $\mathrm{AmB}$ content. The AmB that remained insoluble, and hence not properly incorporated into the nanocarrier, was removed by filtration. The drug concentration in the external aqueous phase of the NE was obtained by ultrafiltration method using a membrane with a weight cut-off of 100,000 (Amicon $^{\circledR}$ - Ultra 4, Millipore, USA). AmB concentration in aqueous phase was found to be negligible. The AmB concentration in NE (before and after filtration) was determined by the validated HPLC method.

The EE of the drug was calculated by the following equation:

$\mathrm{EE} \%=[(\mathrm{AmB}$ before filtration $-\mathrm{AmB}$ after filtration $) / \mathrm{AmB}$ before filtration $]$ $\times 100$

\subsubsection{Assay of AmB by high performance liquid chromatography (HPLC)}

The AmB concentration in NE was evaluated by reverse phase HPLC method. A Waters liquid chromatograph coupled with a 515 model Waters pump, 717 plus automatic injector, and a 2487 model UV detector at $405 \mathrm{~nm}$ were used. A ACE ${ }^{\circledR} \mathrm{RP}-8$ column $(5 \mu \mathrm{m} ; 250 \times 4.6 \mathrm{~mm})$ was used with a mixture of $20 \mathrm{mM}$ sodium edetate/acetonitrile $\mathrm{pH} 5$ (65:35) as mobile phase at a flow rate of $1.2 \mathrm{~mL} \mathrm{~min}^{-1}$ and $20 \mu \mathrm{L}$ as injection volume. The retention time for AmB was about $7.15 \mathrm{~min}$. The linearity curves were 
performed in the range of $0.5-10 \mu \mathrm{g} / \mathrm{mL}$. Regression equation and linearity $\left(r^{2}\right)$ were $y=19,940+95460 x$ and 0.9961 , respectively. Chromatograms of samples of blank NE revealed that there were no interferences absorbing at $405 \mathrm{~nm}$. The assay was validated to establish selectivity, accuracy $(111 \% \pm 2)$, precision, and limits of detection and quantification. The precision was evaluated through the repeatability test comparing with standard solutions. Statistical analysis showed low values of relative standard deviation (RSD $<5.0 \%$ ) and, therefore, a satisfactory precision. Detection and quantification limits were $0.2 \mu \mathrm{g} / \mathrm{mL}$ and $0.5 \mu \mathrm{g} / \mathrm{mL}$, respectively.

\subsubsection{Electronic absorption and circular dichroism (CD) spectra of AmB-loaded nanocarriers}

UV-visible spectroscopy and circular dichroism (CD) spectra are the discriminatory techniques for identifying the different molecular aggregation states of AmB. AmB-loaded NE with or without STE, the C-AmB (Anforicin ${ }^{\circledR}$ ), and an AmB aqueous solution were analyzed by these techniques. All samples were diluted in distilled water at an AmB concentration of $5.0 \mu \mathrm{g} / \mathrm{mL}$. This concentration is higher than that of $\mathrm{AmB}$ critical aggregation concentration $(1 \mu \mathrm{g} / \mathrm{mL})$ (Egito et al., 2002). The blank NE was also analyzed to eliminate the absorption contribution of any other component different from AmB. The absorption and CD spectra were recorded in the range of $300-450 \mathrm{~nm}$ just after dilution at $25^{\circ} \mathrm{C}$ under a nitrogen atmosphere on a computer-assisted Chirascan ${ }^{\mathrm{TM}}$ spectropolarimeter (Applied Photophysics, UK). Data were obtained using an accumulation of five scans with $1.0 \mathrm{~cm}$ path length quartz cuvettes. All the data were expressed in terms of mdeg.

\subsection{Cytotoxicity and in vitro antileishmanial activity studies}

\subsubsection{Culture of macrophages}

The in vitro studies were carried out using the murine macrophage cell line J774 (ATCC/TIB-67TM). Confluent macrophages were subcultured and maintained at $37{ }^{\circ} \mathrm{C}$ in RPMI 1640 medium under a humidified atmosphere $\left(5 \% \mathrm{CO}_{2}\right)$. All media were supplemented with $20 \%$ fetal calf serum (LGC Biotecnologia, São Paulo, Brazil), $2 \mathrm{mM}$ L-glutamine (Gibco-BRL, New York, USA), $100 \mathrm{IU} /$ $\mathrm{mL}$ penicillin $\mathrm{G}$ potassium (USB Corporation, Ohio, USA), $1 \%$ of $100 \mathrm{mM}$ sodium pyruvate solution (Sigma-Aldrich, Missouri, USA), $1 \%$ of $100 \times$ non-essential amino acid solution (SigmaAldrich, Missouri, USA), and $0.2 \%$ of sodium bicarbonate. The $\mathrm{pH}$ was adjusted to 7.20 .

\subsubsection{Culture of parasites}

Promastigotes of Leishmania (Leishmania) amazonensis (IFLA/BR/ 67/PH8) were maintained at $25^{\circ} \mathrm{C}$ in Grace's insect medium. All media were supplemented with $10 \%$ fetal calf serum, $2 \mathrm{mM}$ L-glutamine, and $100 \mathrm{IU} / \mathrm{mL}$ penicillin $\mathrm{G}$ potassium. The $\mathrm{pH}$ was adjusted to 6.50 .

\subsubsection{Cytotoxicity assays}

To evaluate the cytotoxicity of formulations, an in vitro assay on macrophages was performed. This assay involves the conversion of a tetrazolium salt, 3-(4,5-dimethyithiazol-2-yl)-2,5-diphenyltetrazolium bromide (MTT), into a colored, insoluble formazan product depending on the number of viable cells present. Macrophages from adherent cell line J774 were seeded $\left(5 \times 10^{4}\right.$ cells/well $)$ in a 96 -well flat bottom microplate with $90 \mu \mathrm{L}$ of medium. The cells were allowed to adhere for $30 \mathrm{~min}$ at $37{ }^{\circ} \mathrm{C}$ and $5 \% \mathrm{CO}_{2}$ and were then exposed to $10 \mu \mathrm{L}$ of serial dilutions of formulations (AmBloaded and unloaded NE containing $0 \%, 0.1 \%$ or $0.2 \%$ of STE, and C-AmB Anforicin $\mathrm{B}^{\circledR}$ ) for $24 \mathrm{~h}$. Also included were control groups in which the macrophages were not exposed to any drug. After $24 \mathrm{~h}$, the medium was removed and the cells were further incu- bated at $37{ }^{\circ} \mathrm{C}$ with $100 \mu \mathrm{L}$ of MTT $(0.5 \mathrm{mg} / \mathrm{mL})$ for $4 \mathrm{~h}$. Then, $100 \mu \mathrm{L}$ of sodium dodecyl sulfate $(10 \% \mathrm{w} / \mathrm{v})$ was added to the wells. After $16 \mathrm{~h}$, the reduction of MTT to formazan was quantified by measurement the optical density at $570 \mathrm{~nm}$ using an ELISA plate reader (Molecular Devices). The results were expressed as cell viability percentage relative to control group. The AmB concentration capable of inhibiting $50 \%$ of cell growth was expressed as cytotoxic concentration $\left(\mathrm{CC}_{50}\right)$. The $\mathrm{CC}_{50}$ values were determined from concentration-effect curves using Prism 5.0 (GraphPad Software Inc.). The results were expressed as mean of $\mathrm{CC}_{50} \pm$ standard deviation (SD) of, at least, three independent experiments.

\subsubsection{In vitro activity against intracellular amastigotes}

J774 macrophages were harvested at a density of $1 \times 10^{6}$ cells/ well in 24-well plates containing circular glass cover slips. Macrophages were allowed to adhere for $90 \mathrm{~min}$ at $37^{\circ} \mathrm{C}$ and $5 \% \mathrm{CO}_{2}$, when the medium was replaced by a fresh one. Next, macrophages were infected with $L$. (L.) amazonensis promastigotes at a macrophage-promastigote ratio of $1: 10$ and then incubated at $37^{\circ} \mathrm{C}$ and $5 \% \mathrm{CO}_{2}$ for $3 \mathrm{~h}$. Next, the circular cover glass was washed thrice with PBS ( $\mathrm{pH} 7.20$ ) to remove extracellular promastigotes and then supplemented with complete medium. After $24 \mathrm{~h}$, the medium was replaced by fresh medium containing serial dilutions of formulations [unloaded (blank) NE, AmB-loaded NE containing 0\%, $0.1 \%$, or $0.2 \%$ of STE and Anforicin $\left.\mathrm{B}^{\circledR}\right]$. The untreated infected macrophages were used as the control group. The plates were incubated at $37{ }^{\circ} \mathrm{C}$ and $5 \% \mathrm{CO}_{2}$ for $24 \mathrm{~h}$. Next, the cover slip was removed from the well, washed with PBS (pH 7.20), mounted on glass slides, fixed with methanol, and stained with Giemsa for evaluation. Slides were examined, under immersion objective, to evaluate infectivity. At least 300 cells were counted and the percentage of infected cells and total number of amastigotes were recorded.

The percent inhibition of amastigote growth was calculated to each formulation as follows:

Percent of inhibition $(\%)=100-\left(\frac{\frac{\text { Total amastigotes }}{\text { Total macrophages }} \text { Treatment Group }}{\frac{\text { Total amastigotes }}{\text { Total macrophages }} \text { Control Group }} \times 100\right)$

The AmB concentration capable of inhibit $50 \%$ of parasite growth $\left(\mathrm{IC}_{50}\right)$ was determined for each formulation. The $\mathrm{IC}_{50}$ values were determined from concentration-effect curves using Prism 5.0 (GraphPad Software Inc.). The data were expressed as mean of $\mathrm{IC}_{50} \pm \mathrm{SD}$ of, at least, three independent experiments.

\subsection{Stability studies}

AmB-loaded NE with and without STE were injected into amber glass containers and maintained at $4{ }^{\circ} \mathrm{C}$. Sampling aliquots were withdrawn at $0,30,60,90$ and 180 days after preparation. The following parameters were evaluated: total AmB concentration, EE, particle size, PI and zeta potential.

\subsection{Statistical analysis}

One-way analysis of variance (ANOVA) with Bonferroni's Multiple Comparison Test was used to evaluate differences between means. Differences were considered statistically significant when the $p$ value was less than 0.05 .

\section{Results and discussion}

\subsection{Preparation and characterization of the $N E$}

The influence of the STE concentration $(0 \%, 0.1 \%$ and $0.2 \%)$ on droplet size, PI, zeta potential and EE of drug in AmB-loaded NE was evaluated (Table 1). The EE of AmB in NE was nearly $100 \%$ 
regardless of STE concentration. As previously described by Lance et al. (1995), AmB was successfully incorporated into the system using sodium hydroxide solution, followed by neutralization with hydrochloric acid. The main advantage of using this method is that it allows the production of AmB-loaded NE without the use of organic solvents. Low droplet size $(<200 \mathrm{~nm})$ was obtained for all formulations. In addition, there is a significant reduction $(p<0.05)$ in droplet size when the STE concentration increased. The comparison of NE without STE with those containing this amine at $0.1 \%$ or $0.2 \%$ clearly reveals this decrease in size. All NE presented low PI values. Zeta potential value was negative for $\mathrm{NE}$ without STE and this can be explained by the presence of free fatty acids. At pH 7.0, these fatty acids are in the anionic form, and consequently, the charge is negative. In contrast, zeta potential was positive for NE containing STE $0.1 \%$ and $0.2 \%$, which presented values of $+40 \pm 3 \mathrm{mV}$ and $+53 \pm 2 \mathrm{mV}$, respectively. The positive surface charge of these NE is due to the presence of STE, a cationic lipid.

\subsection{Stability studies}

Data of the present work show that AmB-loaded NE with or without STE was stable revealing that particle size, PI, pH, AmB content, and EE remained constant after 180 days (Table 2). A reduction in zeta potential and $\mathrm{pH}$ values was observed, mainly for NE without STE, and this can be attributed to formation of the fatty acids due to hydrolysis of the MCT, a medium-chain triglyceride. Our previous data showed that the AmB-loaded NE without STE was unstable and STE could be required to improve the long-term stability. In fact, the EE for NE without STE drastically decreased after 30 days, but remained high for NE containing STE (Santos et al., 2012). A possible explanation for these contrasting data may be the presence and stabilizing effect of the cholesterol in the NE of the present work. To investigate the stabilizing role of cholesterol another set of experiments was performed. AmBloaded NE with $(0.25 \%$ and $0.5 \%)$ and without cholesterol (STE was not added) were prepared and their stability was evaluated. The EE for AmB drastically decreased and phase separation was clearly visualized after 30 days for NE with (0.25\%) or without cholesterol (data not shown). In contrast, NE containing $0.5 \%$ cholesterol was stable (Table 2). Therefore, the cholesterol significantly improved the stability of AmB-loaded NE. Previously published data revealed interactions between AmB and cholesterol molecules in different models such as lipid vesicles (Baginski et al., 2002; Gabrielska et al., 2006) and micelles. For micelles containing cholesterol, hydrophobic interaction improved stability providing slow release of AmB (Vakil and Kwon, 2008).

\subsection{Electronic absorption and $C D$}

The CD spectra for different AmB formulations are presented in Fig. 2. The spectra of AmB aqueous solution and mainly for conven-

Table 1

Influence of the STE concentration on the droplet size, PI, zeta potential and EE of AmB-loaded NE.

\begin{tabular}{lccc}
\hline \multirow{2}{*}{ Parameters } & \multicolumn{2}{l}{ Concentration of STE } \\
\cline { 2 - 4 } & $0 \%$ & $0.1 \%$ & $0.2 \%$ \\
\hline Droplet size $(\mathrm{nm})$ & $184 \pm 3$ & $173 \pm 2$ & $169 \pm 0$ \\
PI & $0.13 \pm 0.01$ & $0.14 \pm 0.04$ & $0.11 \pm 0.02$ \\
Zeta potential $(\mathrm{mV})$ & $-46 \pm 2$ & $40 \pm 3$ & $53 \pm 2$ \\
EE $(\%)$ & $98 \pm 1$ & $99 \pm 3$ & $99 \pm 1$ \\
\hline
\end{tabular}

The NE was prepared by the hot homogenization method as described in Section 2 . Composition of NE was: $5 \%$ TCM, $1.5 \%$ polysorbate $80,0.5 \%$ cholesterol, STE $(0 \%$, $0.1 \%$ or $0.2 \%$ ), $0.01 \% \alpha$-tocopherol, $2.25 \%$ glycerol and water q.s. to $100 \%$. tional AmB (Anforicin $B^{\circledR}$ ) showed an intense dichroic doublet centered at about $340 \mathrm{~nm}$, which indicated the presence of selfassociated AmB. In significant contrast, the dichroic signal was virtually absent in the spectra of AmB-loaded NE with or without STE. The data of the intense dichroic signal at $340 \mathrm{~nm}$ for conventional $\mathrm{AmB}$ were in agreement with previously published findings (Jain and Kumar, 2010; Joly et al., 1992). It is well known that this signal is greatly reduced in lipid systems loaded with AmB, suggesting an association of AmB with the lipids (Egito et al., 1996; Silva et al., 2013). This reduction was also described for mixtures of deoxycholate AmB with lipid emulsion for parenteral use and it was attributed to an association between $\mathrm{AmB}$ and phospholipids from emulsion (Egito et al., 2002; Joly et al., 1994).

The UV-visible spectra for different AmB formulations are presented in Fig. 3. The spectra of AmB aqueous solution and Anforicin $B^{\circledR}$ exhibited strong absorbance peaks at $340 \mathrm{~nm}$ and $327 \mathrm{~nm}$, respectively, which are traditionally assigned to self-associated AmB. Moreover, less intense peaks indicating the presence of monomeric AmB were observed at 410, 388, and $365 \mathrm{~nm}$. In significant contrast, modifications in absorption spectra could be observed for AmB-loaded NE. The peak at 340 (or at $327 \mathrm{~nm}$ ) was virtually absent, indicating that self-association of $\mathrm{AmB}$ was reduced in NE, whereas the characteristic peaks of the monomeric form of AmB (at 365, 388 and $415 \mathrm{~nm}$ ) were more intense in comparison with those observed for conventional AmB formulation. The increase of amplitude of the band assigned to AmB self-associated species at the expense of those of monomeric form for conventional AmB was also observed by Egito et al. (2002). For mixture of deoxycholate AmB with lipid emulsion an inverse phenomena was reported and our data for $\mathrm{AmB}$-loaded $\mathrm{NE}$ are in line with these findings. In addition, the appearance of peaks at $350 \mathrm{~nm}$ and $415 \mathrm{~nm}$ for the AmB-loaded NE can be attributed to the interaction between AmB and cholesterol molecules as previously described (Charbonneau et al., 2001).

Our data of UV-visible spectra are in agreement with those obtained in CD and clearly showed that AmB was successfully incorporated into the system. The strong interactions between AmB and surfactant/cholesterol at interface of emulsion nanodroplet probably contribute to reduce the drug release from nanocarrier decreasing free AmB concentration including self-associated form. In fact, Lance et al. (1995) elegantly reported that AmB was localized at interface of a lipid emulsion excluding aqueous and oily phases. In contrast, the release of AmB from Anforicin $B^{\circledR}$ is high, probably due to weak interaction between AmB and deoxycholate, contributing for formation of drug aggregates, the more toxic form of AmB (Brajtburg and Bolard, 1996).

\subsection{Cytotoxicity assay}

First, the cytotoxicity of AmB-loaded and blank NE was investigated. J774 macrophages were incubated with formulations and analyzed for their viability by MTT assay. The influence of STE concentration on the $\mathrm{CC}_{50}$ values of AmB-loaded and blank NE in comparison with conventional $\mathrm{AmB}$ (Anforicin $\mathrm{B}^{\circledR}$ ) is presented in Fig. 4. Interestingly, for formulations without STE, neither AmBloaded NE nor blank NE induced cytotoxicity on J774 macrophages and both presented $\mathrm{CC}_{50}$ values higher than $200 \mu \mathrm{g} / \mathrm{mL}$, which was the highest drug concentration used in this study. In contrast, the data clearly showed that cytotoxicity increased considerably with increasing concentration of STE in NE. When the STE concentration was $0.1 \%$, the $\mathrm{CC}_{50}$ values for AmB-loaded NE and blank NE were $6.3 \pm 0.8$ and $5.1 \pm 2.7 \mu \mathrm{g} / \mathrm{mL}$, respectively (insert Fig. 4). Therefore, AmB-loaded and unloaded NE, both without STE, were approximately 40 -fold less toxic to J774 macrophages than the NE containing STE at $0.1 \%$. Cytotoxicity of NE further increased when STE was used at $0.2 \%$ for both the AmB-loaded and unloaded NE, which 
Table 2

Stability studies of the AmB-loaded NE with and without STE.

\begin{tabular}{|c|c|c|c|c|c|}
\hline Parameters & 1st day & 30th day & 60th day & 90th day & 180th day \\
\hline \multicolumn{6}{|l|}{ NE without STE } \\
\hline Droplet size (nm) & $184 \pm 3$ & $191 \pm 2$ & $195 \pm 3$ & $196 \pm 4$ & $198 \pm 2$ \\
\hline PI & $0.13 \pm 0.01$ & $0.07 \pm 0.02$ & $0.13 \pm 0.01$ & $0.11 \pm 0.03$ & $0.11 \pm 0.05$ \\
\hline Zeta potential (mV) & $-46 \pm 2$ & $-39 \pm 1$ & $-33 \pm 0$ & $-41 \pm 1$ & $-27 \pm 0$ \\
\hline $\mathrm{pH}$ & $7.25 \pm 0.01$ & ND & ND & ND & $6.37 \pm 0.01$ \\
\hline AmB content $(\mathrm{mg} / \mathrm{mL})$ & $1.93 \pm 0.03$ & $2.10 \pm 0.00$ & $2.03 \pm 0.05$ & $2.06 \pm 0.28$ & $1.97 \pm 0.01$ \\
\hline $\mathrm{EE}(\%)$ & $98 \pm 1$ & $90 \pm 2$ & $87 \pm 1$ & $82 \pm 6$ & $95 \pm 2$ \\
\hline \multicolumn{6}{|l|}{ NE with STE (0.2\%) } \\
\hline Droplet size (nm) & $169 \pm 0$ & $167 \pm 3$ & $170 \pm 2$ & $167 \pm 4$ & $166 \pm 3$ \\
\hline PI & $0.11 \pm 0.02$ & $0.12 \pm 0.03$ & $0.11 \pm 0.01$ & $0.11 \pm 0.02$ & $0.08 \pm 0.03$ \\
\hline Zeta potential (mV) & $53 \pm 2$ & $52 \pm 2$ & $38 \pm 4$ & $36 \pm 7$ & $44 \pm 3$ \\
\hline $\mathrm{pH}$ & $7.44 \pm 0.21$ & ND & ND & ND & $6.22 \pm 0.01$ \\
\hline AmB content $(\mathrm{mg} / \mathrm{mL})$ & $1.99 \pm 0.01$ & $2.14 \pm 0.04$ & $2.00 \pm 0.01$ & $1.98 \pm 0.00$ & $1.99 \pm 0.01$ \\
\hline $\mathrm{EE}(\%)$ & $99 \pm 1$ & $87 \pm 0$ & $88 \pm 2$ & $94 \pm 4$ & $89 \pm 0$ \\
\hline
\end{tabular}

ND $=$ non determined.

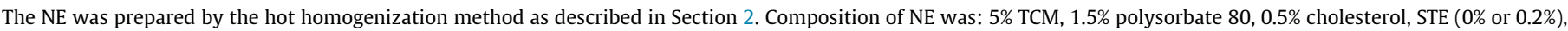
$0.01 \% \alpha$-tocopherol, $2.25 \%$ glycerol and water q.s. to $100 \%$.

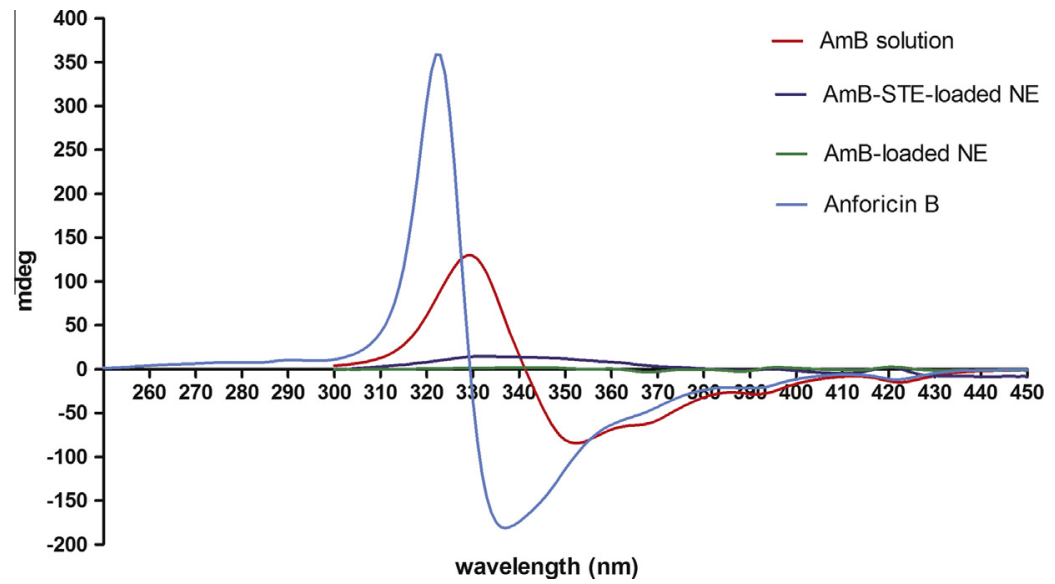

Fig. 2. $C D$ spectra of different formulations of $A m B$ : AmB aqueous solution, Anforicin $B^{\circledR}$, AmB-loaded NE with and without STE.

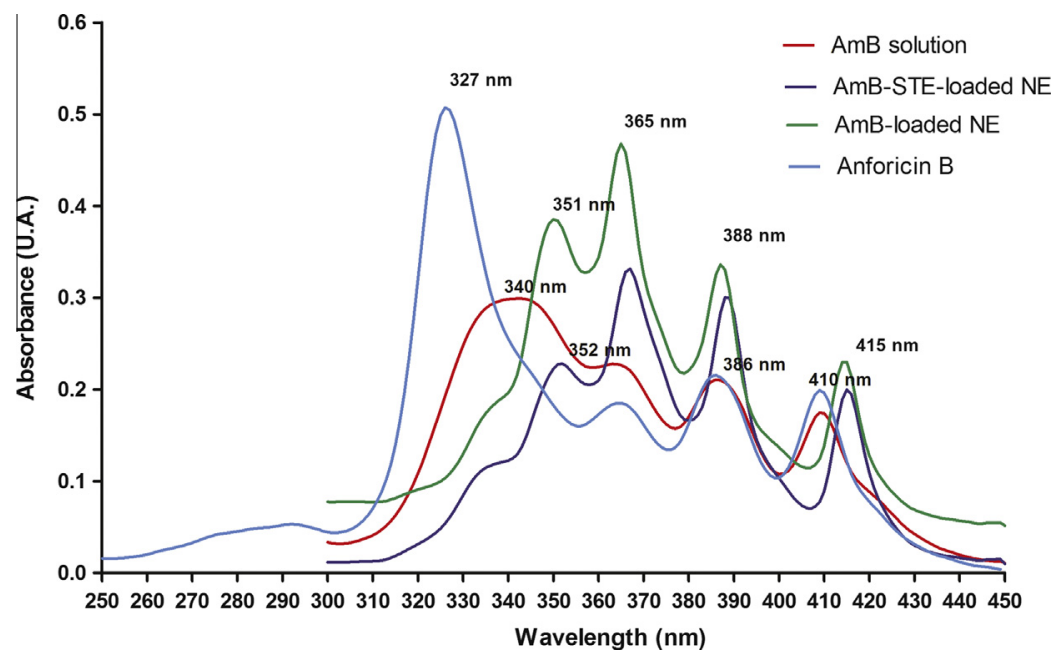

Fig. 3. UV-visible spectra of different formulations of AmB: AmB aqueous solution, Anforicin $\mathrm{B}^{\circledR}$, AmB-loaded NE with (0.1\%) and without STE.

presented $\mathrm{CC}_{50}$ values of $1.8 \pm 0.8 \mu \mathrm{g} / \mathrm{mL}$ and $2.8 \pm 0.8 \mu \mathrm{g} / \mathrm{mL}$, respectively. The presence of $A m B$ has little influence on the cytotoxicity of NE. The cytotoxicity of the NE containing STE could be attributed to the presence of this cationic lipid. These data are in agreement with previous findings, which revealed relatively high cytotoxicity of STE-stabilized lipid emulsions (Hung et al., 2005). The proposed mechanisms of the cytotoxicity are the electrostatic interactions of these cationic amphiphilic molecules with the anionic components of the cell membrane leading to membrane damage (Schöler et al., 2001). 


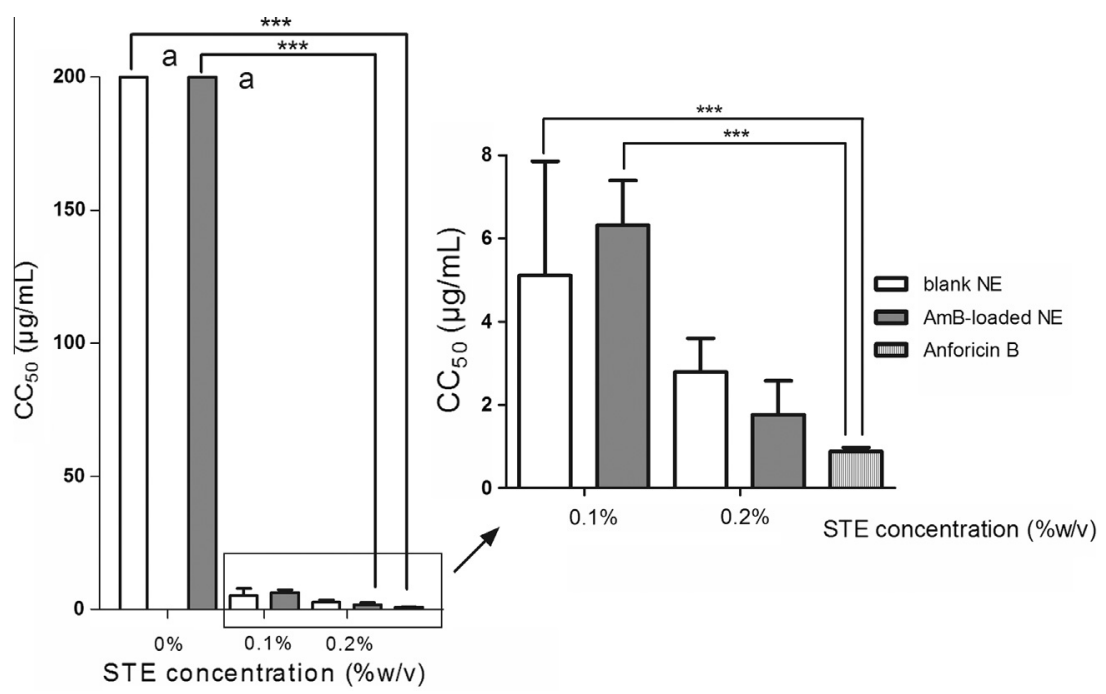

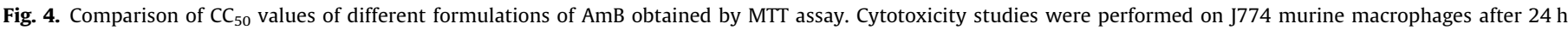

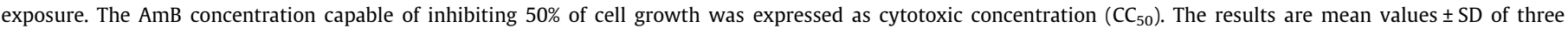
independent experiments.

Comparing the data for AmB-loaded NE and conventional AmB $\left(\right.$ Anforicin $^{\circledR}$ ), it can be clearly observed that the NE-induced toxicity toward macrophages was significantly lower than that presented by the conventional formulation of AmB (insert Fig. 4). These differences could be observed for both AmB-loaded or unloaded NE with STE. It is noteworthy that the $\mathrm{CC}_{50}$ value for conventional AmB formulation was approximately 200 times lower than that observed for AmB-loaded NE without STE. These findings are consistent with previous observations, which showed that the cytotoxic effects of conventional AmB were higher than those observed for AmB lipid carriers. The differences in toxicity of the different lipid formulations depend of the rate at which they AmB release (Brajtburg and Bolard, 1996). For lipid emulsions loaded with $A m B$ as well as for others lipid carriers this toxicity has been attributed to the self-associated free AmB (Egito et al., 2002; Jain and Kumar, 2010; Joly et al., 1994). Our cytotoxicity data are in line with these findings and they are consistent with the results of UV-visible spectroscopy and $C D$ spectra obtained in present study, which showed that NE contained much less selfassociated AmB than conventional AmB.

\subsection{In vitro efficacy against intracellular amastigotes}

The data concerning the influence of the STE concentration ( $0 \%$ and $0.1 \%$ ) on the antileishmanial activity against intracellular amastigotes for AmB-loaded NE are presented in Fig. 5. The data for conventional AmB (Anforicin B ${ }^{P}$ are also shown. The $\mathrm{IC}_{50}$ value for the AmB-loaded NE without STE $(0.21 \pm 0.03 \mu \mathrm{g} / \mathrm{mL})$ was significantly higher than that observed for AmB-STE-loaded NE $(0.11 \pm 0.03 \mu \mathrm{g} / \mathrm{mL})$. These data indicated that the STE enhanced the activity of the AmB since the AmB-STE-loaded NE presented superior efficacy compared to the NE without STE. These findings are in agreement with the data previously published, which suggested that the antileishmanial activity of the STE could contribute synergistically with AmB (Banerjee et al., 2008; Dey et al., 2000). The Anforicin ${ }^{\circledR}$ was more active than AmB-loaded NE, but the differences between the AmB-STE-loaded NE and Anforicin ${ }^{\circledR}$ were not significant.

In general, deoxycholate $A m B$ has proven to be consistently more active in vitro than lipid-based formulations in treating Leishmania infected macrophages (Iman et al., 2011; Yardley and Croft, 1997, 2000). This has been attributed to rapid release of mono-

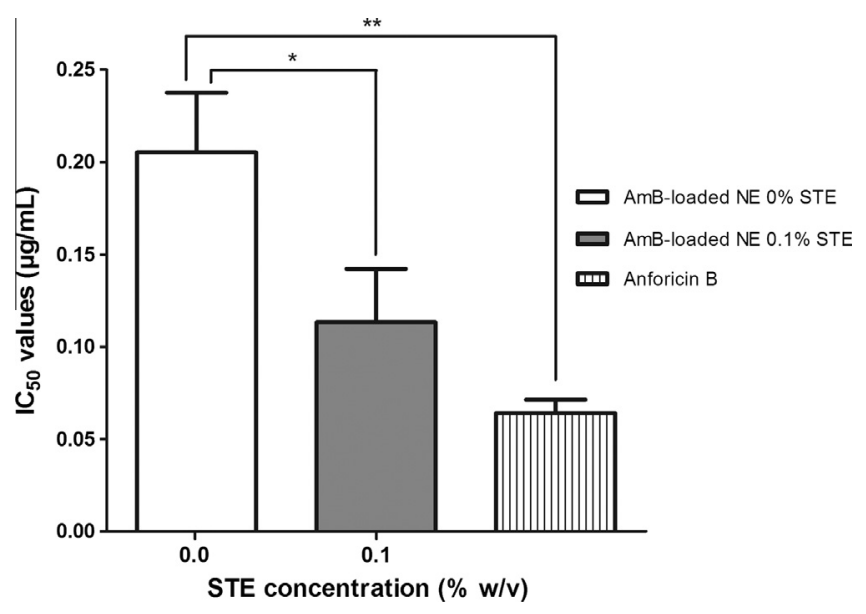

Fig. 5. Comparison of $\mathrm{IC}_{50}$ values of different formulations of $\mathrm{AmB}$. In vitro activity studies were performed against intracellular $L$. L. amazonensis amastigotes after $24 \mathrm{~h}$ exposure. The AmB concentration capable of inhibit $50 \%$ of parasite growth was expressed as $\mathrm{IC}_{50}$. The results are mean values $\pm \mathrm{SD}$ of three independent experiments.

meric drug from conventional $\mathrm{AmB}$ enabling the $\mathrm{AmB}$ to bind to low density lipoproteins (LDL) and be taken up by infected macrophages (Yardley and Croft, 2000). Our data are consistent with these findings, but the presence of STE described in present study enhanced the antileishmanial activity of AmB-loaded NE and represents an interesting alternative.

To evaluate further the potential of these formulations, the selectivity index (SI) was determined from the ratio of cytotoxicity $\left(\mathrm{CC}_{50}\right)$ to antileishmanial activity $\left(\mathrm{IC}_{50}\right)$. The data for $\mathrm{CC}_{50}, \mathrm{IC}_{50}$, and SI for AmB-loaded NE with or without STE and conventional AmB are presented in Table 3. The greater this ratio, the more selective the formulation against the parasite. It is noteworthy that the SI value for NE with and without STE was approximately 17 and 60 times higher than that observed for conventional AmB. These data suggest that such AmB-loaded NE is much more selective in discriminating between the cells of the parasite and those of the host.

Taken together, our data clearly showed that it was possible to incorporate $\mathrm{AmB}$ in $\mathrm{NE}$ using a simple, cheap and easy method 
Table 3

Comparison of the $\mathrm{CC}_{50}, \mathrm{IC}_{50}$ and SI values for the AmB-loaded NE with STE $(0.1 \%)$, AmB-loaded NE without STE and conventional AmB (C-AmB).

\begin{tabular}{lclr}
\hline Formulations & $\mathrm{CC}_{50}{ }^{\mathrm{a}} \pm \mathrm{SD}(\mu \mathrm{g} / \mathrm{mL})$ & $\mathrm{IC}_{50}{ }^{\mathrm{b}} \pm \mathrm{SD}(\mu \mathrm{g} / \mathrm{mL})$ & \multicolumn{1}{c}{$\mathrm{SI}$} \\
\hline NE without STE & $>200$ & $0.21 \pm 0.03$ & $>952$ \\
NE with STE $(0.1 \%)$ & $6.3 \pm 0.8$ & $0.11 \pm 0.03$ & 57 \\
C-AmB & $1.0 \pm 0.1$ & $0.06 \pm 0.01$ & 16 \\
\hline
\end{tabular}

a Cytotoxicity studies were performed on J774 murine macrophages after $24 \mathrm{~h}$ exposure. The AmB concentration capable of inhibiting $50 \%$ of cell growth was expressed as cytotoxic concentration $\left(\mathrm{CC}_{50}\right)$.

b In vitro activity studies were performed against intracellular amastigotes after $24 \mathrm{~h}$ exposure. The AmB concentration capable of inhibit $50 \%$ of parasite growth was expressed as $\mathrm{IC}_{50}$. Data for both $\mathrm{CC}_{50}$ and $\mathrm{IC}_{50}$ were expressed as mean \pm standard deviation (SD) of three independent experiments.

confirming previous findings described by Lance et al. (1995). However, we extended these studies showing that the NE was stable with no degradation or dissociation of AmB from nanocarrier after 180 days. Finally, the NE developed in the present study showed more advantages as carrier of AmB for the treatment of leishmaniasis than the conventional AmB due mainly to reduced cytotoxicity and similar efficacy making it a more attractive alternative for the treatment of leishmaniasis.

\section{Conclusion}

NE loaded with AmB and STE, which enhances the antileishmanial activity, was designed and evaluated. AmB-loaded NE with small particle size $(<200 \mathrm{~nm}$ ), high encapsulation (almost $100 \%$ ) and stability, which could be maintained for at least 180 days, was obtained. In vitro cytotoxicity and antileishmanial activity studies showed that both efficacy and cytotoxicity of AmB-loaded NE increased with increasing STE concentration. However, the AmB-loaded NE was less toxic than the conventional AmB formulation and this can be attributed to reduction of self-associated AmB in lipid nanocarrier as observed in UV-visible spectroscopy and $C D$ studies. These findings suggest that AmB-loaded NE formulation is a promising alternative for the treatment of leishmaniasis.

\section{Conflict of interest}

The authors state no conflict of interest and have received no payment in preparation of this manuscript. All authors have approved the final article.

\section{Acknowledgments}

This work was supported by the "Minas Gerais State Agency for Research and Development" (FAPEMIG, Brazil), and by the Brazilian Agencies CAPES and CNPq. The authors wish to thank Glenn Hawes from the American Language Program of the University of Georgia for editing this manuscript.

\section{References}

Baginski, M., Resat, H., Borowski, E., 2002. Comparative molecular dynamics simulations of amphotericin B-cholesterol/ergosterol membrane channels. Biochim. Biophys. Acta 1567, 63-78.
Banerjee, A., De, M., Ali, N., 2008. Complete cure of experimental visceral leishmaniasis with amphotericin B in stearylamine-bearing cationic liposomes involves down-regulation of IL-10 and favorable T cell responses. J. Immunol. 181, 1386-1398.

Brajtburg, J., Bolard, J., 1996. Carrier effects on biological activity of amphotericin B. Clin. Microbiol. Rev. 9, 512-531.

Charbonneau, C., Fournier, I., Dufresne, S., Barwicz, J., Tancrède, P., 2001. The interactions of amphotericin B with various sterols in relation to its possible use in anticancer therapy. Biophys. Chem. 91, 125-133.

Desjeux, P., 2004. Leishmaniasis: current situation and new perspectives. Comp. Immunol. Microbiol. Infect. Dis. 27, 305-318.

Dey, T., Anam, K., Afrin, F., Ali, N., 2000. Antileishmanial activities of stearylaminebearing liposomes. Antimicrob. Agents Chemother. 44, 1739-1742.

Egito, E.S.T., Fessi, H., Appel, M., Barratt, G., Legrand, P., Bolard, J., Devissaguet, J.-P.h., 1996. A morphological study of an amphotericin B emulsion-based delivery system. Int. J. Pharm. 145, 17-27.

Egito, E.S.T., Araújo, I.B., Damasceno, B.P.G.L., Price, J.C., 2002. Amphotericin B/ emulsion admixture interactions: an approach concerning the reduction of amphotericin B toxicity. J. Pharm. Sci. 91, 2354-2366.

Gabrielska, J., Gagos, M., Gubernator, J., Gruszecki, W., 2006. Binding of antibiotic amphotericin B to lipid membranes: a ${ }^{1} \mathrm{H}$ NMR study. FEBS Lett. 580, $2677-$ 2685.

Hung, C.-F., Hwang, T.-L., Chang, C.-C., Fang, J.-Y., 2005. Physicochemical characterization and gene transfection efficiency of lipid emulsions with various co-emulsifiers. Int. J. Pharm. 289, 197-208.

Iman, M., Huang, Z., Szoka Jr., F.C., Jaafari, M.R., 2011. Characterization of the colloidal properties, in vitro antifungal activity, antileishmanial activity and toxicity in mice of a distigmasterylhemisuccinoyl-glycero-phosphocholine liposome-intercalated amphotericin B. Int. J. Pharm. 408, 163-172.

Jain, J.P., Kumar, N., 2010. Development of amphotericin B loaded polymersomes based on (PEG) $)_{3}$-PLA co-polymers: factors affecting size and in vitro evaluation. Eur. J. Pharm. Sci. 20, 456-465.

Joly, V., Bolard, J., Saint-Julien, L., Carbon, C., Yeni, P., 1992. Influence of phospholipid/amphotericin B ratio and phospholipid type on in vitro renal cell toxicities and fungicidal activities of lipid-associated amphotericin B formulations. Antimicrob. Agents Chemother. 36, 262-266.

Joly, V., Farinotti, R., Saint-Julien, L., Chéron, M., Carbon, C., Yeni, P., 1994. In vitro renal toxicity and in vivo therapeutic efficacy in experimental murine cryptococcosis of amphotericin B (Fungizone) associated with Intralipid. Antimicrob. Agents Chemother. 38, 177-183.

Lance, M.R., Washington, C., Davis, S.S., 1995. Structure and toxicity of amphotericin B/triglyceride emulsion formulations. J. Antimicrob. Chemother. 36, 119-128.

Larabi, M., Yardley, V., Loiseau, P.M., Appel, M., Legrand, P., Gulik, A., Bories, C. Croft, S.L., Barratt, G., 2003. Toxicity and antileishmanial activity of a new stable lipid suspension of amphotericin B. Antimicrob. Agents Chemother. 47, 3774-3779.

Muller, R.H., Schmidt, S., Buttle, I., Akkar, A., Schimitt, J., Bromer, S., 2004. SolEmuls ${ }^{\circledR}$ - novel technology for the formulation of i.v. emulsions with poorly soluble drugs. Int. J. Pharm. 269, 293-302.

Pal, S., Ravindran, R., Ali, N., 2004. Combination therapy using sodium antimony gluconate in stearylamine-bearing liposomes against established and chronic Leishmania donovani infection in BALB/c mice. Antimicrob. Agents Chemother. 48, 3591-3593.

Santos, C.M., de Oliveira, R.B., Arantes, V.T., Caldeira, L.R., de Oliveira, M.C., Egito, E.S.T., Ferreira, L.A.M., 2012. Amphotericin B-loaded nanocarriers for topical treatment of cutaneous leishmaniasis: development, characterization, and in vitro skin permeation studies. J. Biomed. Nanotechnol. 8, 322-329.

Schöler, N., Olbrich, C., Tabatt, K., Muller, R.H., Hahn, H., Liesenfeld, O., 2001. Surfactant, but not the size of solid lipid nanoparticles (SLN) influences viability and cytokine production of macrophages. Int. J. Pharm. 221, 57-67.

Silva, A.E., Barratt, G., Chéron, M., Egito, E.S.T., 2013. Development of oil-in-water microemulsions for the oral delivery of amphotericin B. Int. J. Pharm. 454, 641648

Torrado, J.J., Espada, R., Ballesteros, M.P., Torrado-Santiago, S., 2008. Amphotericin B formulations and drug targeting. J. Pharm. Sci. 97, 2405-2425.

Vakil, R., Kwon, G.S., 2008. Effects of cholesterol on the release of amphotericin B from PEG-phospholipid micelles. Mol. Pharm. 5, 98-104.

Vyas, S.P., Gupta, S., 2006. Optimizing efficacy of amphotericin B through nanomodification. Int. J. Nanomed. 1, 417-432.

Yardley, V., Croft, S.L., 1997. Activity of liposomal amphotericin B against experimental cutaneous leishmaniasis. Antimicrob. Agents Chemother. 41, $752-756$.

Yardley, V., Croft, S.L., 2000. A comparison of the activities of three amphotericin B lipid formulations against experimental visceral and cutaneous leishmaniasis. Int. J. Antimicrob. Agents 13, 243-248. 